\title{
Quantifiers and the Foundations of Quasi-Set Theory
}

\author{
Jonas R. BECKer AREnhart \\ DÉCIO KRAUSE \\ Federal University of Santa Catarina
}

\begin{abstract}
In this paper we discuss some questions proposed by Prof. Newton da Costa on the foundations of quasi-set theory. His main doubts concern the possibility of a reasonable semantical understanding of the theory, mainly due to the fact that identity and difference do not apply to some entities of the theory's intended domain of discourse. According to him, the quantifiers employed in the theory, when understood in the usual way, rely on the assumption that identity applies to all entities in the domain of discourse. Inspired by his provocation, we suggest that, using some ideas presented by da Costa himself in his seminars at UFSC (the Federal University of Santa Catarina) and by one of us (DK) in some papers, these difficulties can be overcome both on a formal level and on an informal level, showing how quantification over items for which identity does not make sense can be understood without presupposing a semantics based on a 'classical' set theory.
\end{abstract}

Keywords: Quasi-sets, semantic of non-classical systems, logic of quantum mechanics.

Dedicated to Prof. Newton da Costa for his 80th birthday and for his inspiration in so many philosophical questions.

\section{Introduction}

One of the challenges that appear in the development of non-classical logics is related to the semantics of such systems. Generally speaking, it is recognized that a semantics conduced according to the standard methods employ as metalanguage some one of the 'classical' set theories, such as ZF (the Zermelo-Fraenkel set theory, generally encompassing the axiom of choice). Such a semantics comprises some immediate difficulties on the foundational side so that, from a philosophical point of view, it would seems to be not completely absent of a priori commitments (given by such meta-framework). Saying in brief, since standard ZF is itself based on classical logic, it seems to be already committed to the very principles of this logic, the same ones whose avoidance was the main driving force behind the proposal of a non-classical system. ${ }^{1}$ Thus, instead of liberating us from the classical principles, a semantics to a non-classical system, if grounded in ZF (and the standard mathematical frameworks in general), commits us again to classical logic, viz., that one

Principia 13(3): 251-68 (2009).

Published by NEL — Epistemology and Logic Research Group, Federal University of Santa Catarina (UFSC), Brazil. 
assumed in the metalanguage. Alternatively, in a homier way, as put by da Costa, Béziau, and Bueno, "[o]ne reintroduces, so to speak, by the backdoors, exactly what was intended to be left on the entrance!" (da Costa et al. 1995, 44). ${ }^{2}$

A specific case in which this problem can be clearly seen is related to the consideration of non-reflexive logics (see da Costa \& Krause 1994, 1997; French \& Krause 2006, chaps.7 and 8). ${ }^{3}$ One of the main motivations for the use of these systems come from the supposition that some items in the intended domain of discourse lack identity conditions. For instance, it is sometimes assumed that non-relativistic quantum mechanics can be seen as providing us a world with such kind of entities. If we assume a metaphysical hypothesis like this one, it seems that, at least from a philosophical point of view, we should have an adequate non-classical logic to deal with these entities ('entities without identity', which may be indistinguishable but without turning to be the same object-identical). Based on such motivations, a particular type of non-reflexive set theory was developed, strongly enough to encompass a mathematics suitable for physics, termed quasi-set theory. This theory aims at to pursue seriously the motto inherited from H. Post (Post 1963), namely, that the indiscernibility (or indistinguishability) of quantum objects should be attributed to these entities right from the start, so as in recognizing (following Schrödinger) that neither identity nor difference would make sense to some entities (namely, the 'elementary particles'). ${ }^{4}$ Usually, the way of dealing with indiscernible things within 'standard' frameworks is by assuming certain symmetry conditions, which mask the logical fact that every entity that obeys classical logic is, in a certain sense, an individual; for a detailed discussion, see French \& Krause 2006. These collections, called quasi-sets, are so that, despite the impossibility of identification of its elements, they do have a cardinal number, which intuitively speaking expresses how many elements there is in the considered quasi-set. ${ }^{5}$ Furthermore, the theory encompasses a weaker relation of indistinguishability that holds among items of the same type, which enables us to express that elements may be indistinguishable (indiscernible), although not identical (see the following sections for a sketch of quasi-set theory).

In his seminars at UFSC and through particular conversations, da Costa has expressed doubts about the possibility of explaining how quantifiers are used in quasiset theory for, in principle, quantification would make sense only when the concept of identity can be applied to the considered objects. According to da Costa, a nonclassical logic, in order to be a legitimate rival of classical logic, would encompass a semantics which conforms to its basic assumptions, that is, a semantics in which the main motivations of the considered logic are maintained (see da Costa et al. 1995). So, he claims, if one cannot make sense of quantifiers without presupposing identity, then one cannot claim that a logic without identity is a suitable system of logic for dealing, for instance, with indistinguishable elementary particles, typical of non-relativistic quantum mechanics.

Principia 13(3): 251-68 (2009). 
In what concerns quasi-set theory, since its language involves quantification, then, in accordance with such ideas, in order to make statements which intuitively mean, say, 'every element of the quasi-set $x$ is so and so', or 'there is an element in $x$ which is so and so', we should be able to explain how do we grasp the meaning of such sentences without presupposing that identity is valid for the items under quantification. In particular, if we want to show that we can really do that, we must do that without implicitly assuming that our metalanguage is a classical set theory like $\mathrm{ZF}$, even though it is kept on an informal level only. The problem, then, amounts in finding an explanation of the semantical basis for the quasi-set theory's underlying logic. Certainly, from these discussions it follows that the underlying logic of $\mathfrak{Q}$ is not classical logic, whose semantics is a classical one, and it must be some kind of non-reflexive logic, in order to preserve our motivations.

For some, it would seem that a classical metalanguage is necessary to make intelligible the usual reading of the quantifiers, for according to this view, such a reading is not possible without an overall validity of the concept of identity. They could argue that when we say, for example, 'there is some $x$ such that $x$ is $P$ ', this should be taken to mean 'there is a set that is the extension of $P$, to which the denotation of $x$ belongs', leading us back to set theory. If this kind of reasoning was correct, then any reasonable meaning of the quantifiers would be tied inevitably to identity and, then, by employing quantifiers in quasi-set theory, we would reintroduce identity for the items under discussion through the metalanguage, causing us to have to abandon the very project we began with. To deal properly with these questions, we should state adequately the underlying logic and the metalanguage we are using to study quasi-set theory. Our aim here is to show that a reasonable understanding of the quantifiers exist, so that we can avoid the risk of re-introducing identity for every object in the domain of the discourse.

Our procedure will be based in the classical case, really, it will be a generalization of it. So, let us take a brief look at those parts of the usual approach which are relevant for our purposes. Consider, for example, quantification in ZF. How do we make clear and mathematically precise our intuitive understanding of the quantifiers used by the underlying logic in this case? We rely, in general, on the Tarskian semantics for first order languages, assuming that the underlying logic of ZF is (say) classical first order logic with identity. This semantics, it is claimed, gives us a mathematization of the intuitive meaning of the quantifiers. First-order logic, besides its axioms, has its specific semantics, and both contribute to make clear the meaning of the logical constants, including the quantifiers.

Now, Tarskian semantics is generally stated using informal set theory, and if pressed to make our assumptions clear, we say that we are relying on ZF, the same ZF which is being taken here as our case study, whose underlying logic is (by hypothesis) exactly the first-order logic whose semantical understanding is in question! This 
is not a case of circularity, as it becomes clear when we make the simple distinction between the meta-theory in which we work and the object theory we are interested in. In this case, both are taken to be ZF. This case is an example of the 'demand of coherence' imposed generally on non-classical logics, as stated above, that the metalanguage should not commit us with principles deviant from those granted by the very logic under study. So, ZF is used to state and clarify the meaning of logical constants for the system of logic which is its own underlying logic, as we shall see below. One possible way to go out of such an assumption, if there is one, seems to suppose that the syntax of the formal system alone is able to clarify the meaning of the terms of the language through the axioms and rules of inference (in a strict formal way, as suggested for instance by Haskell B. Curry (Curry 1951). But then, one must face the problem that the same set of axioms can be used to ground systems of logic that could be different when semantic considerations entered the discussion, that is, some systems of logic may differ only in the semantics presented, having the same formal apparatus when viewed from syntactic point of view alone. We will not follow this 'syntax only' strategy here. On the contrary, we shall be concerned with semantical issues, for they are of course in the core of the discussions regarding the ontological commitments of theories.

Thus, our strategy will be the same one used in classical logic. The underlying logic of $\mathfrak{Q}$ will have its semantic based in quasi-set theory itself, giving the adequate meaning to its logical constants. We first recover very briefly how this is done for classical first order logic, roughly speaking, by mapping the language in set theory and then with this theory (ZF) as the background language, it is possible to design a formal semantics for the language of first order logic. Obviously, our claim is that the choice of the metalanguage always carries some commitments that drive the semantics we are considering. This shows that we can in fact study the axiomatics of first-order ZF within ZF proper, although we cannot prove that there is a model of this theory within ZF (due to the second Gödel's incompleteness theorem). In our specific case, we show how to do such a thing for the language of the underlying $\operatorname{logic}$ of the object theory $\mathfrak{Q}$, this time translating it to our metatheory $\mathfrak{Q}$, where the adequate semantic meaning of the logical symbols can be done yet, we insist, we cannot prove that there is a model of $\mathfrak{Q}$ within $\mathfrak{Q}$ (supposed consistent). Any charge of circularity here would be rightly directed to the classical case, for the procedure is really a generalization of that case, and we take as an easy matter to avoid the problem of an adequate distinction between the object language and the metalanguage.

\section{The 'classical' case}

To make our paper self-contained, we recall briefly in this section the procedure described in Ebbinghaus et al. 1994, 113-4). There, it is shown how a system of first Principia 13(3): 251-68 (2009). 
order classical logic can be developed inside ZF, so that both its semantics and syntax are made using a classical metalanguage (namely, that of ZF proper). Besides an enumerable quantity of variables $x_{1}, x_{2}, \ldots, x_{n} \ldots$, we take as logical symbols the connectives $\neg$ and $\rightarrow$, the universal quantifier $\forall$, and the equality sign $=$. For simplicity we restrict ourselves to a language $\mathfrak{L}$ with predicate symbols $P^{1}, P^{2}, \ldots$ as non-logical constants only, where $P^{n}$ denotes an $n$-ary predicate symbol, and different predicates of the same weight can be distinguished by a sub-index, but we won't consider them here.

We begin by showing how the symbols of the language can be seen as certain sets. The variables are identified with the elements of $\omega$, vis., the set of natural numbers within ZF, following von Neumann's standard definition. So, we assume, for example, that the variable $x_{n}$ is identified with the natural number $n$. The other logical symbols are defined as follows: $\neg$ is associated to the ordered pair $\langle 0,0\rangle, \rightarrow$ to the pair $\langle 0,1\rangle, \forall$ is identified with $\langle 0,2\rangle$, while $=$ with $\langle 0,3\rangle$. For the non logical symbols, we represent each predicate $P^{n}$ with $n \neq 0$ by the pair $\langle 1, n\rangle$.

Now, let us consider the case of atomic formulas. In our language, they come in two versions: in the first case, they are formulas of the form $x_{n}=x_{m}$, which can be seen as ordered triples $\langle n,=, m\rangle$, with $n, m \in \omega$. All the atomic formulas of this type constitute the set $A t^{=}$which by definition is the set $\omega \times\{=\} \times \omega$, where ' $\times$ ' denotes the cartesian product of sets. The second case is constituted by formulas of the form $P^{n} x_{i_{0}} \ldots x_{i_{n-1}}$, which are taken to be pairs such as $\left\langle P^{n}, f\right\rangle$, where $f$ is a function from $n$ (which is a set) to $\omega$, so that, for example, if $f$ is such that $f(0)=2$ and $f(1)=6$, then $\left\langle P^{2}, f\right\rangle$ is the formula $P^{2} x_{2} x_{6}$. The set of all such formulas, $A t^{P}$ is by definition the set $\left\{\left\langle P^{n}, f\right\rangle: n \in \omega\right.$ and $\left.f: n \mapsto \omega\right\}$.

Thus, the set $\mathfrak{F}$ of formulas of $\mathfrak{L}$ is the smallest set satisfying the following conditions:

1. $A t^{=} \cup A t^{P} \in \mathfrak{F}$;

2. if $\alpha \in \mathfrak{F}$, then $\langle\neg, \alpha\rangle \in \mathfrak{F}$;

3. if $\alpha, \beta \in \mathfrak{F}$, then $\langle\alpha, \rightarrow, \beta\rangle \in \mathfrak{F}$;

4. if $n \in \omega$ and $\alpha \in \mathfrak{F}$, then $\langle\forall, n, \alpha\rangle \in \mathfrak{F}$.

Now, following the usual methods (Ebbinghaus et al., loc.cit.), one can develop a formal semantics for this language. Since this is supposed to be well known, we will not recall the details here. Our point is to emphasize the general technique, for later we want to enlighten the similarities between this 'classical' case and quasi-set theory. What really matters is that, since the theory used to establish this standard semantics is classical set theory, there are some features of the semantics due to this assumption that cannot be overlooked from a philosophical and foundational point 
of view. The crucial assumption, in this case, at least in what concerns our purposes, is that identity makes sense to every entity in the domain of quantification.

\section{Outlining quasi-set theory}

In this section, we give an outline of the most basic notions of quasi-set theory that play an important role in the discussion that follows (for further details, see French \& Krause 2006, chap.7). This is not the place to present all the postulates and definitions of the theory, so we just revise the main ideas and results which are important for what follows. Intuitively speaking, a quasi-set is a collection of objects such that some of them may be indistinguishable without turning out to be identical. Of course this is not a strict 'definition' of a quasi-set, acting more or less as Cantor's 'definition' of a set as "any collection into a whole $M$ of definite and separate [that is, distinguishable] objects $m$ of our intuition or our thought" (see the discussion in French \& Krause 2006, §6.4), serving just to provide an intuitive account of the concept.

The quasi-set theory $\mathfrak{Q}$ has in its main motivations some considerations taken from quantum physics, mainly in considering Schrödinger's idea that the concept of identity do not make sense when applied to elementary particles (Schrödinger 1952, 17-8; as remarked earlier, he considered just non-relativistic quantum mechanics). Another motivation is (in our opinion) the need, stemming from philosophical worries, of dealing with collections of absolutely indistinguishable items that need not be the same ones. ${ }^{6}$ Of course, viewed from a formal point of view, $\mathfrak{Q}$ can also be developed independently of any intended interpretation, but here we shall always keep in mind this 'quantum' motivation since, after all, it is the intended interpretation that has originated the problem of the development of the theory.

The first point is to guarantee that identity and indistinguishability (or indiscernibility) will nor collapse into one another when the theory is formally developed. We assume that identity (symbolized by '=') is not a primitive relation, but the theory has a weaker concept of indistinguishability (symbolized by ' $\equiv$ ') instead. This is just an equivalence relation and holds among all objects of the considered domain. If the domain is divided up into objects of two kinds, the $m$-objects (standing for 'microobjects') and $M$-objects (for 'macro-objects') and quasi-sets of them (and probably having other quasi-sets as elements as well), then identity (defined as so that it has all the properties of standard identity of ZF) can be defined for $M$-objects and quasisets having no $m$-objects in their transitive closure (this concept is like the standard one). Thus, if we take just the part of theory obtained by ruling out the $m$-objects and collections (quasi-sets) having them in their transitive closure, we get a copy of ZFU (ZF with Urelemente); if we further eliminate the $M$-objects, we get a copy of the 'pure' ZF.

Principia 13(3): 251-68 (2009). 
Technically, expressions like $x=y$ are not always well formed, for they are not formulas when either $x$ or $y$ denote $m$-objects. We express that by saying that the concept of identity does not make sense for all objects (it should be understood that this is just a way of speech). The objects (the $m$-objects) to which the defined concept of identity does not apply are termed non-individuals for historical reasons (see French \& Krause 2006). As a result (from the axioms of the theory), we can form collections of $m$-objects which have no identity-in this sense; these collections may have a cardinal (termed its 'quasi-cardinal') but not an associated ordinal. Thus, the concept of ordinal and of cardinal are independent, as in some formulations of ZF proper. So, informally speaking, a quasi-set of $m$-objects is such that its elements cannot be identified by names, counted, ordered, although there is a sense in saying that these collections have a cardinal (which cannot be defined by means of ordinals, as usual-but see below).

It is important to remark that, when $\mathfrak{Q}$ is used in connection with quantum physics, the $m$-objects are thought of as representing quantum entities (henceforth, q-objects), but they are not necessarily 'particles' in the standard sense-associated with classical physics or even with orthodox quantum mechanics; waves, field excitations (the 'particles' in quantum field theory-QFT) or perhaps even strings can be taken as possible interpretations of the $m$-objects, although this needs qualification. Generally speaking, whatever 'objects' sharing the property of being indistinguishable can also be values of the variables of $\mathfrak{Q}$ (see Falkenburg 2007, chap.6 for a survey of the various different meanings that the word 'particle' has acquired in connection with quantum physics).

Another important feature of $\mathfrak{Q}$ is that standard mathematics can be developed using its resources, for the theory is conceived in such a way that ZFU (and hence also ZF, perhaps with the axiom of choice, ZFC) is a subtheory of $\mathfrak{Q}$. In other words, the theory is constructed so that it extends standard Zermelo-Fraenkel with Urelemente (ZFU); thus standard sets (of ZFU) can be viewed as particular qsets (that is, there are qsets that have all the properties of the sets of ZFU, and the objects in $\mathfrak{Q}$ corresponding to the Urelemente of ZFU are identified with the $M$-atoms of $\mathfrak{Q}$ ). The 'sets' in $\mathfrak{Q}$ will be called $\mathfrak{Q}$-sets, or just sets for short. To make the distinction, the language of $\mathfrak{Q}$ encompasses a unary predicate $Z$ such that $Z(x)$ says that $x$ is a set. It is also possible to show that there is a translation from the language of ZFU into the language of $\mathfrak{Q}$ so that the translations of the postulates of ZFU are theorems of $\mathfrak{Q}$; thus, there is a 'copy' of ZFU in $\mathfrak{Q}$, and we refer to it as the 'classical' part of $\mathfrak{Q}$. In this copy, all the usual mathematical concepts can be stated, as for instance, the concept of ordinal (for the $\mathfrak{Q}$-sets). This 'classical part' of $\mathfrak{Q}$ plays an important role in the formal developments of the next sections.

Furthermore, it should be recalled that the theory is constructed so that the relation of indiscernibility, when applied to $M$-atoms or $\mathfrak{Q}$-sets, collapses into standard 
identity (of ZFU). The $\mathfrak{Q}$-sets are qsets whose transitive closure (defined as usual) does not contain $m$-atoms (in other words, they are 'constructed in the classical part of the theory—see Fig. 1).

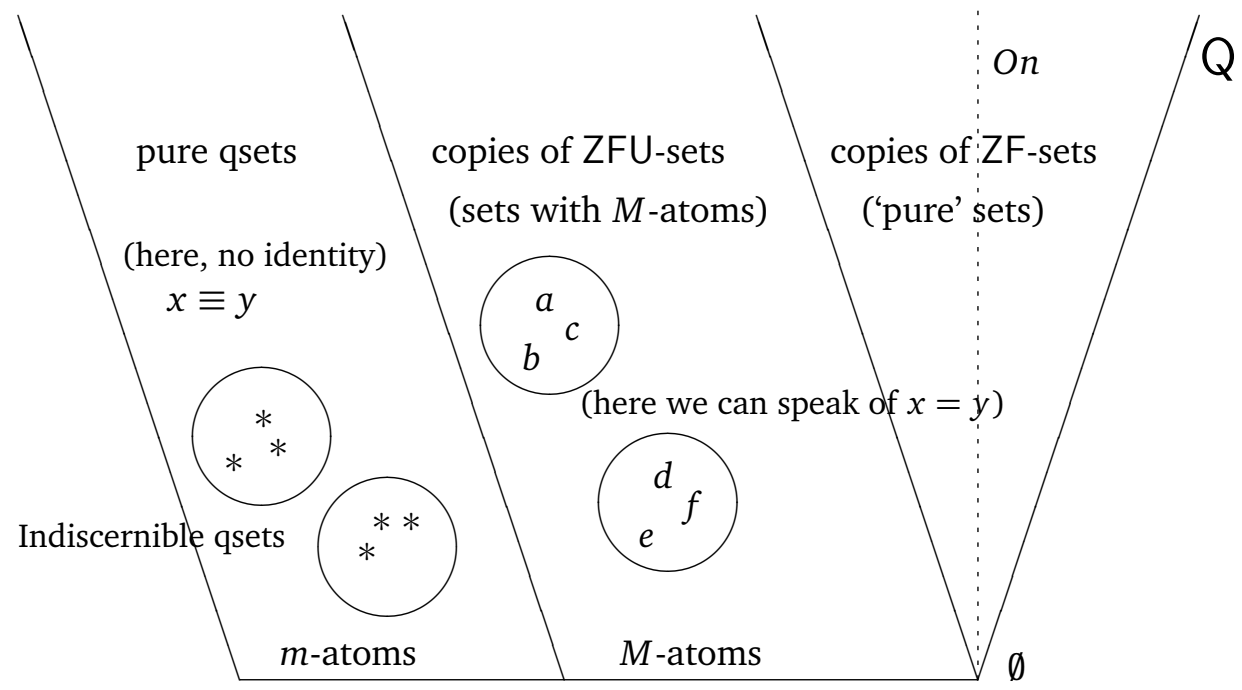

Figure 1: The Quasi-Set Universe Q: On is the class of ordinals, defined in the classical part of the theory.

In order to distinguish between $\mathfrak{Q}$-sets and qsets that may have $m$-atoms in their transitive closure, we write (in the metalanguage) $\{x: \varphi(x)\}$ for the former and $[x: \varphi(x)]$ for the latter. In $\mathfrak{Q}$, we term 'pure' those qsets that have only $m$-objects as elements (although these elements may be not always indistinguishable from one another, that is, the theory is consistent with the assumption of the existence of different kinds of $m$-atoms-that is, not all of them must be indiscernible from one another), and to them it is assumed that the usual notion of identity cannot be applied (that is, let us recall, $x=y$, as well as its negation, $x \neq y$, are not well formed formulas if either $x$ or $y$ stand for $m$-objects). Notwithstanding, the primitive relation $\equiv$ applies to them, and it has the properties of an equivalence relation.

The concept of extensional identity, as said above, is a defined notion, and it has the properties of standard identity of ZFU. More precisely, we write $x={ }_{E} y$ (read ' $x$ and $y$ are extensionally identical') iff they are both qsets having the same elements (that is, $\forall z(z \in x \leftrightarrow z \in y)$ ) or they are both $M$-atoms and belong to the same qsets (that is, $\forall z(x \in z \leftrightarrow y \in z)$ ). From now on, we shall not bother to always write $=_{E}$, using simply the symbol "=" for the extensional equality, as we have done above.

Since $m$-atoms are to stand for entities which cannot be labeled, for they do not enter in the relation of identity, it is not possible in general to attribute an ordinal 
to collections whose elements are denoted by $m$-atoms. As a consequence, for these collections it is not possible to define the notion of cardinal number in the usual way, that is, through ordinals. ${ }^{7}$ In the version of the theory we shall be considering, to remedy this situation, we admit also a primitive concept of quasi-cardinal which intuitively stands for the 'quantity' of objects in a collection. ${ }^{8}$ The axioms for this notion grant that certain quasi-sets $x$ (in particular, those whose elements are $m$ objects) may have a quasi-cardinal, written $q c(x)$, even when it is not possible to attribute an ordinal to them.

To link the relation of indistinguishability with qsets, the theory also encompasses an 'axiom of weak extensionality', which states (informally speaking) that those quasi-sets that have the same quantity (expressed by means of quasi-cardinals) of elements of the same sort (in the sense that they belong to the same equivalence class of indistinguishable objects) are indistinguishable by their own. One of the interesting consequences of this axiom is related to the quasi-set version of the non observability of permutations, which is one of the most basic facts regarding indistinguishable quanta (for a discussion on this point, see French \& Rickles 2003). In brief, remember that in standard set theories, if $w \in x$, then of course $(x-\{w\}) \cup\{z\}=x$ iff $z=w$. That is, we can 'exchange' (without modifying the original arrangement) two elements iff they are the same elements, by force of the axiom of extensionality. In contrast, in $\mathfrak{Q}$ we can prove the following theorem, where $[[z]]$ (and similarly $[[w]])$ stand for a quasi-set with quasi-cardinal 1 whose only element is indistinguishable from $z$ (respectively, from $w$ ) -the reader shouldn't think that this element is identical to either $z$ or $w$, for the relation of equality doesn't apply to these items; the set theoretical operations can be understood according to their usual definitions):

Theorem: [Unobservability of Permutations] Let $x$ be a finite quasi-set such that $x$ does not contain all indistinguishable from $z$, where $z$ is an $m$-atom such that $z \in x$. If $w \equiv z$ and $w \notin x$, then there exists $[[w]]$ such that

$$
(x-[[z]]) \cup[[w]] \equiv x .
$$

The theorem works to the effect that, supposing that $x$ has $n$ elements, then if we 'exchange' their elements $z$ by corresponding indistinguishable elements $w$ (set theoretically, this means performing the operation $(x-[[z]]) \cup[[w]])$, then the resulting quasi-set remains indistinguishable from the one we started with. In a certain sense, it does not matter whether we are dealing with $x$ or with $(x-$ $[[z]]) \cup[[w]]$. So, within $\mathfrak{Q}$, we can express that 'permutations are not observable', without necessarily introducing symmetry postulates, and in particular to derive 'in a natural way' the quantum statistics (see Krause et al. 1999; French \& Krause 2006, chap.7). Further applications to the foundations of quantum mechanics can be seen in Domenech et al. 2008.

Principia 13(3): 251-68 (2009). 


\section{Formal semantics: the case of $\mathfrak{Q}$}

From now on, we shall be working in the classical part of $\mathfrak{Q}$ (that is, we use it as our metatheory), which was shown in the above section to be that part of $\mathfrak{Q}$ in which classical mathematics can be developed, and which deals with what we call the 'classical entities' of the theory. Our goal is to show how the syntax of firstorder theories can be developed in $\mathfrak{Q}$, and in particular, the syntactical part of $\mathfrak{Q}$ itself, (formulas and symbols should be understood as classical items) when $\mathfrak{Q}$ is also taken as the object language. We will not go into the details when they can be recovered from our previous discussion for the classical case.

Once again, the individual variables of the theory are to be represented by the set $\omega$, which exists in the classical part of $\mathfrak{Q}$. For the case of the logical symbols, as before $\neg$ is the pair $\langle 0,0\rangle, \rightarrow$ is $\langle 0,1\rangle$, and $\forall$ is $\langle 0,3\rangle$. We can go on and define the non-logical vocabulary for first-order theories, just by considering how predicates can be taken to be certain quasi-sets as we did before in the case of classical logic, taking them to be some specific sets. Our goal now is to consider the particular case of the non-logical vocabulary of $\mathfrak{Q}$ itself, considered as an object theory.

For $m, M$ and $Z$, which are unary predicate symbols of $\mathfrak{Q}$, we define respectively the quasi-sets $\langle 1,1\rangle,\langle 2,1\rangle$ and $\langle 3,1\rangle$. Here, the second number indicate the rank of the symbol, while the first one indicates the particular enumeration of the symbol. This is the general reasoning behind the attribution. For the binary relations $\equiv$ and $\in$ we define $\langle 1,2\rangle$ and $\langle 2,2\rangle$, respectively. Finally, for the functional symbol $q c$ we attribute $\langle 4,1\rangle$.

As before, the quasi-sets of formulas can now be defined but, since now we have a functional symbol $q c$ as an additional term along with variables, we must say what we mean by a quasi-set of terms. As said above, the variables are the elements of $\omega$. For the terms of the form $q c\left(x_{n}\right)$, we take the quasi-set $[\langle q c, n\rangle: n \in \omega]$. The terms are the elements of the q-set $\mathfrak{T}$ defined as $\omega \cup[\langle q c, n\rangle: n \in \omega]$. For easy reference, we denote an arbitrary element of $\mathfrak{T}$ by $t_{n}$.

Thus we have in our particular case atomic formulas of the type $t_{n} \equiv t_{m}$, which as before are seen as triples, but now with $\equiv$ replacing $=$, that is, $\left\langle t_{n}, \equiv, t_{m}\right\rangle$. The atomic formulas of this type constitute the q-set $A t^{\equiv}$, which by definition is the quasiset $\mathfrak{T} \times\{\equiv\} \times \mathfrak{T}$. Formulas of the form $m t_{n}, Z t_{n}, M t_{n}$, and $t_{n} \in t_{m}$ are particular cases of formulas of the general form $P^{n} x_{i_{0}} \ldots x_{i_{n-1}}$. As before, each of them is taken to be a pair $\left\langle P^{n}, f\right\rangle$, where $f$ is a quasi-function from $n$ (the rank of the symbol in question) to $\mathfrak{T}$, so that, for example, if $f$ is such that $f(0)=\langle q c, n\rangle$ and $f(1)=2$, then $\langle\in, f\rangle$ is the formula $q c\left(x_{n}\right) \in x_{2}$. The quasi-set of all such formulas, $A t^{P}$ is by definition $\left[\left\langle P^{n}, f\right\rangle: n \in \omega\right.$ and $\left.f: n \mapsto \mathfrak{T}\right]$.

With these details explicated, the quasi-set $\mathfrak{F}$ of formulas of quasi-set theory turns to be the smallest quasi-set satisfying the conditions: 
1. $A t^{\equiv} \bigcup A t^{P} \in \mathfrak{F}$;

2. if $\alpha \in \mathfrak{F}$, then $\langle\neg, \alpha\rangle \in \mathfrak{F}$;

3. if $\alpha, \beta \in \mathfrak{F}$, then $\langle\alpha, \rightarrow, \beta\rangle \in \mathfrak{F}$;

4. if $n \in \omega$ and $\alpha \in \mathfrak{F}$, then $\langle\forall, n, \alpha\rangle \in \mathfrak{F}$.

\section{The underlying logic of $\mathfrak{Q}$ and its semantics}

Let us call the first-order underlying logic of $\mathfrak{Q}$ 'the logic of indiscernibility', $\mathscr{L}_{\mathfrak{Q}}$. Its language has the usual logical connectives $\neg, \wedge, \vee$ and $\rightarrow$ (one needs to assume only $\neg$ and $\rightarrow$ as primitive, the other ones being defined as usual), a denumerable set of individual variables $x_{1}, x_{2}, \ldots$, and a binary predicate symbol $\equiv$. Specific non-logical symbols can be added, as for instance, in the case of $\mathfrak{Q}$, the unary predicate symbols $m, M$ and $Z$, the binary predicate symbol $\in$ and the unary functional symbol $q c$. Terms and formulas are defined as usual.

The postulates of $\mathscr{L}_{\mathfrak{Q}}$ are the following ones:

1. A complete set of postulates for first-order classical logic without identity.

2. The postulates of indiscernibility, namely:

(a) $\forall x(x \equiv x)$

(b) $\forall x \forall y(x \equiv y \rightarrow y \equiv x)$

(c) $\forall x \forall y \forall z(x \equiv y \wedge y \equiv z \rightarrow x \equiv z)$

It is clear that any equivalence relation models $\equiv$, in particular, standard identity $=$ models it. But the most interesting case is when we can speak of indiscernible but not identical things, and this can be done within standard systems (such as ZFC) only if we restrict the discourse to a particular structure or consider just a few primitive predicates and relations so that the considered structure has other automorphisms than the identity function. But since in ZFC we can always extend a structure to an structure having only the identity mapping as its automorphism, the talk of indiscernible things in ZFC turns to be quite artificial. The better way, to do it, in our opinion, is to employ an adequate mathematical theory, such as $\mathfrak{Q}$.

Let's recover for one moment what we are pursuing with the constructions just presented. The first point is that, since the very presentation of the syntax of the language of $\mathscr{L}_{\mathfrak{Q}}$ involves some set theoretical apparatus, like a family of variables and the use of recursion on sets of formulas, we don't need to commit ourselves to classical set theoretical apparatus and all that comes with it. We can confine 
ourselves to a classical part of $\mathfrak{Q}$ and grant that we are not taking everything to be classical sets, in particular, not everything in the intended range of quantification must be an individual, when the intended interpretation of the symbols come into consideration.

The situation is analogous to the formulation of classical set theory. One starts with some sets of symbols and, by applying the usual procedures, we can develop a formal theory of set theory. So, if one wants to argue that quasi-set theory necessitates a classical theory to be formulated, for the very syntax presupposes classical concepts, we can respond to this objection by showing that it indeed needs some classical concepts, but these can come from a quasi-set theory which is being in this case used as metalanguage.

The second and most important point of interest for our purposes is that since $\mathfrak{Q}$ has a first-order underlying logic $\mathscr{L}_{\mathfrak{Q}}$, the foregoing construction helps us in avoiding falling in the trap mentioned in the beginning of our paper. There we mentioned that a non-classical logic becomes committed, through the metalanguage, with the very classical principles it meant to avoid, if its semantics is developed in ZFC or other classical set theory. Now we recall that the underlying logic of quasi-set theory must not be simply classical first-order logic without identity, for the semantics for these logics are made in ZFC, as we have exemplified above with the case of an equivalence relation modeling $\equiv$. So, despite the fact that the axioms were the same as those presented for first-order classical logic, the semantics of $\mathscr{L}_{\mathfrak{Q}}$ is based in quasi-set theory, allowing for non-individuals in the range of quantification. This kind of semantics is a generalization of Tarskian semantics, and it is in fact the specific case presented in French \& Krause 2006, chap.8.

To insist a little more on this topic, the underlying logic of $\mathfrak{Q}$, when considered from the syntactical point of view, is a first order language without identity, whose axioms are the same as those used for classical logic (see the presentation of $\mathscr{L}_{\mathfrak{Q}}$ above). But the intended semantics for this logics differs from the specific one for classical logic. When providing the semantics for $\mathscr{L}_{\mathfrak{Q}}$, we allow that not only sets can be used in the domain of quantification, but also quasi-sets, so that non-individuals also may appear there. It is a matter of generalization of the usual case, having the classical semantics as a limit case.

Again, to avoid confusion, it is important to distinguish between the theory we are studying and the theory we are using to develop these matters. We use quasiset theory to clarify the semantics of a first-order logic $\mathscr{L}_{\mathfrak{Q}}$. By the nature of its semantics, $\mathscr{L}_{\mathfrak{Q}}$ is a non-classical logic, more specifically, it is a kind of non-reflexive logic, since it permits that objects for which neither identity nor difference applies appear in the domain of quantification, and it is exactly the logic underlying the formal system of quasi-set theory $\mathfrak{Q}$ which we investigate, the object theory. So, through these devices we can show how to develop the underlying logic of $\mathfrak{Q}$ without 
having to re-introduce individuality for every item in the domain of quantification, and granting that the first-order theories that employ this logic as underlying logic can 'talk' about non-individual in a (supposed) philosophically satisfying way (see Krause 2008).

The discussion we have advanced relies on the fact that a system of logic can be said to acquire its characterization not only through its syntax, but also through its semantics (by the way, this is Newton da Costa's position too). As we said, one should not assume from the outset that the reading of the formulas is based on classical set-theoretical semantics, which would commit us with a classical ontology, but one should choose the adequate framework for the semantical developments, namely, $\mathfrak{Q}$.

The point that a classical semantics for these logics would commit us with a kind of classical ontology is another thesis of philosophical relevance. In general, a system of logic (of first or higher order, or set theories) carries with it some metaphysical implications. Classical logic, in particular, takes it to be the case that the world is composed of atomic facts, in which, for example, laws like the excluded middle holds (this resembles Russell and Wittgenstein's logical atomism) and, in particular, what is relevant for our discussion, it takes identity to be everywhere applicable. These features are also encapsulated in the framework in which the semantics is formulated, so, as we would say it, it describes a world of individuals. When we want to discuss the problem of indiscernible particles in quantum physics, for example, and hold that they are not individuals, failing to satisfy the axioms for identity of classical logic, then these commitments should be avoided, and our logic must cope with our philosophical position, as we hold that quasi-set theory may do that.

In fact, using $\mathfrak{Q}$ as the 'background' theory in Quine's sense (Quine 1969), we can commit ourselves with non-individuals in such a way that we can propose the negation of one of Quine's most famous mottos, namely, that there are no entities without identity, for we can, with the resources of $\mathfrak{Q}$, argue that non-individuals can be values of variables as well, so we can state our maxim: there are entities without identity (for details, see Krause 2008). This in particular shows that da Costa's generalization of Quine's dictum that to be is to be the value of a variable, by adding (contrary to Quine), "of a certain language and subjected to a certain underlying logic", we should also add and modulo a given background theory used as its metalanguage for, as we have seen, depending on this theory (for instance, taking $\mathfrak{Q}$ ), we can be committed to non-individuals in our ontology. Of course this point should be investigated further. 


\section{Further Philosophical Discussion}

An additional relevant point in the philosophical discussion of semantics for formal languages is the extent to which we can say that a Tarski-style like semantics really elucidates the meaning of the logical terms of the considered language. We are aware of the difficulties involved in such discussions, and do not aim at to dispute the fact that such a semantics serves mainly as a mathematical procedure to make rigorous and sharp some intuitive understanding of the assumed concepts. This intuitive understanding guides the definitions adopted in the semantics, as it may be seen from a valuation semantics for the classical propositional calculus. The fact is that it is of course debatable if the formal semantics really captures the relevant aspects of the informal use of the considered concepts.

So, one may still be not satisfied with the technical developments of the last section and the use we have made of it to justify the primitive concepts of the theory $\mathfrak{Q}$. Sure, one may still ask for the explication of the intuitive basis underlying those developments. The dissatisfaction perhaps can be expressed in the following lines: "It is all right to proceed that way for classical logic, for the formal semantics is only a mean to write in a mathematical fashion some of our informal understanding of the logical terms. In the case of quasi-set theory, one ought to provide also an informal understanding, otherwise not much is really gained through the proposed formal semantics."

We think that these objections can be overcome, in some sense, since the axioms of quasi-set theory can also be seen as written to cope with some intuitive features of our use of the language in contexts in which we can take identity as a meaningless concept. We think that in some contexts it is possible to consider identity as a derived notion, and not a primitive one, and in these contexts it may be seen as 'parasitical' one in relation to other more basic notions such as indistinguishability and cardinality. Really, identity can be understood in terms of these last ones, and thus, taken as a convenient fiction useful for practical purposes. From an epistemological point of view, Hume may be read as a precursor of this view, obviously not understanding identity as relying on cardinality. Really, if we are free to suggest that those who have questioned the intuitive concept of identity, or of its applicability, then David Hume can in fact be regarded as a forerunner of non-reflexive logics. Obviously without any commitment to logical systems as we understand them today, Hume has questioned the applicability of identity in the re-identification processes, as it is clear in his Treatise (Hume 1985). For an analysis of this idea and of its possible relationships with Schrödinger, see Becker \& Krause 2006.

We take it to be the case that, for practical purposes, and not only in contexts where indiscernible particles are concerned, identity does not need to be taken as a primitive concept, for it is enough to assume a weaker notion of indistinguishability

Principia 13(3): 251-68 (2009). 
(as in quasi-set theory, where it is, as we have seen, a congruence relation). With indistinguishability, one obtains identity as a derived notion, which we can apply when dealing with everyday objects, by taking its negation, discernibility, to be understood as difference. That is, for usual things, we take that discernibility acts as a fake individuation principle, and we make no claim that it neither really individuates only one object, nor that it ascribes a kind of primitive thisness to it. That is, we do not go beyond those assumption that are convenient for practical purposes. So, language works as usual, with names and quantification, for these items, and everything works as if they were individuals (some of these ideas are a modification of some proposals that were first advanced by Teller 1998).

Secondly, to understand the quantifiers in an informal sense, when they are applied to the intuitive aggregates of non-individuals, we don't need to use identity, for we can resort to a distinguishing feature of such aggregates: the cardinal number. When we say something such as 'all items are so and so', we can make a paraphrase and consider it to mean 'All $m$ of them are such and such', the $m$ belonging to a suitable qset. Difference (the negation of identity), at least within these contexts involving indistinguishable items, can be derived from the cardinality talk. Taking the cardinals as having primacy, difference is then a derived by-product, for we may assume that, since there are 'more than one item', they may be thought as being distinct, although this kind of talk might be not expressed in the object language. Anyway, the 'cardinal talk' does not imply difference. Just for a simple example, let us consider a collection with two indistinguishable members (for instance, two bosons in the same state). We can say (in the metalanguage) that they are different, not because we present some distinctive feature (they really do not present any), but because they are two, and the difference should be taken not as the negation of usual identity, but just as an abbreviation of speech.

Our claim is that one can consistently maintain that all these features are captured in our previous discussion on the semantics of the underlying logic of $\mathfrak{Q}$. This is one of the possible ways to give and intuitive semantics to quantifiers when we consider that we are dealing with aggregates that might have a quasi-set theoretical behavior. Our tentative proposal of basing the intuitive understanding of quantifiers and even the usual reference mechanisms for classical objects, is a first approach on the subject, but we think there is still much to be said in favor of such an approach, and we shall work on the details in future papers.

\section{Conclusion}

In this paper we were concerned with a semantics for a particular kind of nonreflexive logic. Our suggestion was that we can employ quasi-set theory to help us

Principia 13(3): 251-68 (2009). 
to understand quantification over non-individuals, following a generalization of the usual procedure employed for classical logic semantics. The domain of quantification can be a quasi-set, and the usual definitions used for classical logic can be extended to the case under consideration.

From an informal point of view, we related the issue to a suggestion that nonindividuals may be metaphysically prior to individuals, and that identity (and difference) may be seen as 'derived' from indistinguishability and cardinality. Working on this possibility, one can see that quantification and naming can be recovered for what we have called the 'standard (or usual) objects', and applies for items in general. This is a first approach to the subject, but we think that it shows that nonreflexive logics, in particular quasi-set theory, may be relevant in this context for it seems to be on a par with the specific type of certain metaphysical claims (specifically, a metaphysics of non-individuals), helping us to articulate rigorously a general view on such particulars. ${ }^{9}$

\section{References}

Becker, J. \& Krause, D. 2006. Hume, Schrödinger e a individuação de objetos físicos. Revista Eletronica Informação e Cognição 5(2): 59-71.

Curry, H. B. 1951. Outlines of a Formalist Philosophy of Mathematics. Amsterdam: NorthHolland Publishing Company.

da Costa, N. C. A.; Béziau, J. Y.; Bueno, O. 1995. What is semantics? A brief note on a huge question. Sorites-Electronic Quarterly of Analytical Philosophy 3: 43-7.

da Costa, N. C. A. \& Krause, D. 1994. Schrödinger Logics. Studia Logica 53(4): 533-50.

- 1997. An intensional Schrödinger logic. Notre Dame Journal of Formal Logic 38(2): 179-94.

Devlin, K. 1993. The Joy of Sets: Fundamentals of Contemporary Set Theory. New York: Springer-Verlag.

Domenech, G. \& Holik, F. 2007. A Discussion on Particle Number and Quantum Indistinguishability. Foundations of Physics 37(6): 855-78.

Domenech, G.; Holik, F.; Krause, D. 2008. Q-spaces and the foundations of quantum mechanics. Foundations of Physics 38(11): 969-94.

Ebbinghaus, H. D.; Flum, J.; Thomas, W. 1994. Mathematical Logic. New York: SpringerVerlag.

Falkenburg, B. 2007. Particle Metaphysics: A Critical Account of Subatomic Reality. Berlin: Springer.

French, S. \& Krause, D. 2006. Identity in Physics. A historical, philosophical, and formal analysis. Oxford: Oxford Un. Press.

French, S. \& Rickles, D. P. 2003. Understanding Permutation Symmetry. In K. Brading and E. Castellani (eds.) Symmetries in Physics: New Reflections. Cambridge: Cambridge University Press, 212-38.

Hume, D. 1985. Treatise of Human Nature. 2nd ed. L. A. Selby-Bigge. Oxford: Oxford Un. Press.

Principia 13(3): 251-68 (2009). 
Jammer, M. 1974. The Philosophy of Quantum Mechanics. New York: John Wiley \& Sons.

Krause, D. 2008. Nota sobre o comprometimento ontológico com não-indivíduos. In R. A. Martins, C. S. Silva, J. M. H. Ferreira e L. A. P. Martins (eds.) Filosofia e História da Ciência do Cone Sul - Seleção de Trabalhos do $5^{\circ}$ Encontro. Campinas: Associação de Filosofia e História da Ciência do Cone Sul, 125-32.

Krause, D.; Sant'Anna, A. S.; Volkov, A. G. 1999. Quasi-set theory for bosons and fermions: quantum distributions. Foundations of Physics Letters 12(1): 51-66.

Post, H. 1963. Individuality and Physics. The Listener, 10 october 1963, pp. 534-7. (reprinted in Vedanta for East and West 132, 1973, pp.14-22.)

Quine, W. V. 1969. Ontological Relativity and Other Essays. New York: Columbia Un. Press.

Schrödinger, E. 1952. Science and Humanism. Cambridge: Cambridge Un. Press.

Teller, P. 1998. Quantum Mechanics and Haecceities. In E. Castellani (ed.) Interpreting Bodies: Classical and Quantum Objects in Modern Physics. Princeton: Princeton University Press, 114-41.

Jonas R. Becker Arenhart and DÉcio Krause Programa de Pós-Graduação em Filosofia Universidade Federal de Santa Catarina Florianópolis, SC Brazil

jonas . becker2@gmail.com deciokrause@gmail.com

Resumo. Neste artigo discutimos algumas questões propostas por Newton da Costa relacionadas aos fundamentos da teoria de quase-conjuntos. Seus questionamentos aqui considerados tratam da possibilidade de uma compreenão semântica da teoria, principalmente devido ao fato de que identidade e diferença podem não ser aplicáveis para algumas das entidades no domínio pretendido da teoria. De acordo com ele, o modo usual de se compreender os quantificadores utilizados na teoria depende da hipótese de que a identidade deve valer para todas as entidades no domínio de discurso. Inspirados pelas suas questões, sugerimos que essas dificuldades podem ser superadas tanto em um nível formal quanto em um nível informal, mostrando como a quantificação sobre itens para os quais a identidade não faz sentido pode ser entendida sem pressupor uma semântica baseada em uma teoria de 'clássica' de conjuntos.

Palavras-chave: Quase-conjuntos, semântica para lógicas não clássicas, lógica da mecânica quântica.

\section{Notes}

${ }^{1}$ Of course there are several distinct formulations of ZF, say in first-order logic or higherorder logic, and even taken one of these approaches, one has several alternatives.

${ }^{2}$ Interesting that the physicist and philosopher C. F. von Waizsäcker expressed similar opinion by means of his 'principle of semantic consistency', according to which "the rules by we

Principia 13(3): 251-68 (2009). 
describe and guide our measurement, defining the semantics of the formalism of a theory, must be in accordance with the laws of the theory" (cited by Jammer 1974, 156).

${ }^{3}$ We call non-reflexive those logics which deviate from the 'traditional theory of identity', in particular, by violating the principle of identity in some of its forms. The paradigmatic case is related to the first-order reflexive law of identity, namely, $\forall x(x=x)$, also called the principle of identity of the first-order classical logic.

${ }^{4}$ Although Schrödinger restricted his discussions to the particles of non-relativistic quantum mechanics, we can extend the discussion to the 'particles' of other quantum theories as well; for an account on such distinctions, see Falkenburg 2007, chap.6.

${ }^{5}$ It should be remembered that according to the standard definition, a cardinal is a particular ordinal, hence it makes sense only under an identification of the considered elements.

${ }^{6}$ This is of course a way of speech.

${ }^{7}$ We just recall that an ordinal is a transitive set which is well-ordered by the membership relation, and that a cardinal is an ordinal $\alpha$ such that for no $\beta<\alpha$ there does not exist a bijection from $\beta$ to $\alpha$. For details of these concepts, see Devlin 1993.

8 The notion of quasi-cardinal can be defined for finite quasi-sets; see Domenech \& Holik 2007.

9 The second author was supported by an individual research grant from The National Council for Scientific and Technological Development (CNPq), process \#304540/2006-4.

Principia 13(3): 251-68 (2009). 